\title{
A Code Optimization Package for REDUCE
}

\author{
J.A.van Hulzen ${ }^{1}$, B.J.A. Hulshof ${ }^{2}$, B.L. Gates ${ }^{3}$ and M.C. van Heerwaarden ${ }^{1}$ \\ ${ }^{1}$ Twente University, Dept. of Comp. Science, P.O. Box 217, 7500 AE Enschede, the Netherlands \\ ${ }^{2}$ Philips Research. Building WB 346, P.O.Box 80.000, 5600 JA Eindhoven, the Netherlands \\ ${ }^{3}$ The Rand Corporation, 1700 Main Street, Santa Monica, CA 90406, U.S.A.
}

\begin{abstract}
A survey of the strategy behind and the facilities of a code optimizaton package for REDUCE are given. We avoid a detailed discusssion of the different algorithms and concentrate on the user aspects of the package. Examples of straightforward and more advanced usage are shown.
\end{abstract}

\section{Introduction}

An important application of computer algebra systems is the generation of code for numerical purposes via automatic or semi-automatic program generation. GENTRAN $[3,4]$, a flexible general-purpose package, was especially developed to assist in such a task, when using MACSYMA or REDUCE.

Attendant to automatic program generation is the problem of automatic source-code optimization. This is a crucial aspect because code generated from symbolic computations often tends to be made up of lengthy arithmetic expressions. One of our test examples contained, for instance, 20534 additions and substractions, 41746 multiplications, 12473 integer exponentiations and 7990 other operations, such as function calls. These lengthy expressions will be grouped together in blocks of straightline code in a program for numerical purposes. The main objective of source-code optimization is to minimize the number of (elementary) arithmetic operations in such blocks. This form of optimization is often helpful in reducing redundancy in expressions. Simplification algorithms applied on expressions viewed as entities, neither guarantee complete structure preservation nor allow improvements inside expressions by renaming sub-expressions. Optimizing compilers ought to deal effectively and efficiently with the average, hand coded program. The enormous, arithmetic intensive expressions, easily producable by a computer algebra system, fall outside the range of the FORTRANprograms, once analysed and discussed by Knuth [17]. He suggested that optimization of the arithmetic in such a program is slightly overdone. This may explain why even in reasonably recent literature, such as [1], optimization of arithmetic code is hardly discussed. The dag-models, usually employed for optimization of arithmetic code, hardly allow the application of any algebraic identity (see for instance [6]). These models often force constants to act as if they were indeterminates and powers
Permission to copy without fee all or part of this material is granted provided that the copies are not made or distributed for direct commercial advantage, the ACM copyright notice and the title of the publication and its date appear, and notice is given that copying is by permission of the Asociation for Computing Machinery. To copy otherwise, or to republish, requires a fee and/or specific permission. as objects requiring function calls, i.e. they force to think of $2 a+3 b$ and $4 a+6 b$ or of $a^{2}, a^{4}$ and $a^{6}$ as being different entities. Our optimization strategy however, requires the validity of some elementary algebraic laws. We employ heuristic techniques to reduce the arithmetic complexity of the given representation of a set of input expressions $E_{i n}$, thus producing a set of output expressions $E_{\text {oxf }}$. The optimized version of the earlier mentioned test example containes "only" 4316 additions and substractions, 4919 multiplications, 13 integer exponentiations and 60 other operations. $E_{\text {in }}$ and $E_{\text {out }}$ define blocks of code, which would compute the same exact values for the same exact inputs, thus implicitly proving the correctness of the underlying software. Obviously the use of $E_{\text {out }}$ saves a considerable amount of execution time in comparison with the application of $E_{i n}$. Johnson e.a. [16] suggest that such transformations do not destabalize the computations. However this is only apparent after a careful error analysis of both $E_{\text {in }}$ and $E_{\text {out }}$. In view of the size of both $E_{\text {in }}$ and $E_{\text {out }}$ such an analysis has to be automatized as well. Although work in this direction is in progress $[12,19]$ it is not the subject of this paper.

The current version of our code optimization package is entirely written in RLISP. It can be used as an extension of REDUCE [9]. It allows to subject almost any set of proper REDUCE assignment statements to a heuristic search for common (sub)expressions (cse's). The output is obtained as a sequence of assignment statements, by default given in REDUCE syntax. The first version of the package was designed to optimize the description of REDUCE-statements, generated by NETFORM $[20,21]$. This version was tailored $t$ a restrictive class of problems, occurring mainly in electrical network theory, thus implying that the righthand sides (rhs's) in the input were limited to elements of $Z_{2}[V]$, where $V$ is a set of identifiers. The second version [15] allowed rhs's from Z [V]. For both versions the validity of the commutative and the associative law was assumed. A third version evolved from the latter package by allowing to apply the distributive law, i.e. by replacing (sub)expressions like $a . b+a . c$ by $a .(b+c)$ whenever possible. But the range of possible applications of this version was really enlarged by redefining $\mathrm{V}$ as a set of kemels, implying that, at least by that time, almost any proper REDUCE expression could function as a rhs. The mathematical capabilities of this version are shortly summarized in [22], in the context of code generation and optimization for 
finite-element analysis. It is used in combination with GENTRAN, for the construction of Jacobians and Hessians [11] and also in experiments with strategies for code vectorization [5]. The user-interface of the present version relies on some GENTRAN facilities.

In $[14,15]$ the optimization-strategy was characterized as a composite function $R^{-1} \circ T^{\circ} R$. The function $R$ defines the parsingprocess, used to store the input in matrix-structures. Such an incidence matrix representation of a set of arithmetic expressions is a two-dimensional structure where the rows represent expresssion or sub-expression references and the columns represent indentifier references such as variable and function names. $A$ given sub-expression will be entered in one of two types of incidence matrices, one for sums and one for products, depending on the nature of the arithmetic operation at the top level of the expression. The two matrices are correlated by auxillary predecessor-successor information at the row level for every sub-expression reference. The actual entries in the matrices are either multiplicative numerical coefficients for the sums matrix or powers for the products matrix. The inverse function $R^{-1}$ defines the output-production. The function $T$ defines the optimization process itself. It essentially consists of a heuristic remodelling of the (extendable) matrices in combination with storing information required for a fast retrieval and correct insertion of the detected cse's in the output. This is accomplished by an iteratively applied search, resulting in a stepwise reduction of the arithmetic complexity of the input set, using an extended version of Breuer's grow factor algorithm $[2,14,15]$. It is applied until no further profit is gained, i.e. until the reduction in arithmetic complexity stops. Before producing output, a finishing touch can be performed to further reduce the arithmetic complexity with some locally applied techniques. The overall process can be summarized as follows:

$$
\begin{aligned}
& R: E_{0} \rightarrow\left(D_{0}, \text { profit }_{0}\right) \\
& T_{\beta}:\left(D_{i}, \text { profit }_{i}\right) \rightarrow\left(D_{i+1}, \text { profit }_{i+1}\right), i=0, \ldots, \lambda-1 . \\
& F:\left(D_{\lambda},\right. \text { profit } \\
& ) \rightarrow D_{\lambda} \\
& R^{-1}: D_{\lambda} \rightarrow E_{\lambda}
\end{aligned}
$$

$D_{0}$ is the data structure, created as a result of the parsing step defined by $R$ and performed on the input $E_{0}$. The termination condition depends on some profit criterium related to the arithmetic complexity of the latest version of the input. $D_{i}$. Hence we assume profit $_{i}=$ true for $i=0, \ldots, \lambda-1$ and profit $t_{\lambda}=$ false. The function $T$ is composite as well, and defined by $T=F^{\circ} T_{\beta}{ }^{\lambda} \cdot T_{\beta}$ defines one iteration step, i.e one application of the extended version of Breuer's algorithm. The function $F$ defines a finishing touch, resulting in the final version $D_{\lambda}$ of $D_{0}$, used to produce the output $E_{\lambda}$. We discuss $T$ in more detail in section 2.

Input-parsing was originally based on the assumption that simplification was either already performed or still had to be carried out. When input was delivered in simplified form, for instance when read from a file, prefix-form parsing was required. Simplification of input before parsing it, demanded a standardquotient-form parsing. Both altcrnatives forced to intervene in the normal REDUCE command interpretation. Similar arguments hold for the last stage in the process. Here we produce intermediate output, being an association-list, called prefixlist, and formed by pairs $\left(\mathrm{lhs}_{i}\right.$. rhs $\mathrm{s}_{\mathrm{i}}$ ), for $\mathrm{i}=1(1)$ length(prefixlist). The output, is produced as a sequence of assignment statements $\mathrm{TL}\left(\mathrm{lh} s_{\mathrm{i}}=\mathrm{rh} \mathrm{s}_{\mathrm{i}}\right.$ ), for $\mathrm{i}=1$ (1)lenght(prefixlist), where TL is a function, designed to translate its argument into a user-defined Target Language.
The present version makes use of some GENTRAN facilities to translate its input into LISP prefix-forms. These forms are acceptable by that part of the parser we used for already simplified input in earlier versions.

Another advantage is that the optimization package itself is now immune to effects of future changes in REDUCE, assuming GENTRAN remains maintained adequately. A problem might be that coefficient-domain specific data have to rely on GENTRAN for their translation into the desired prefix-form. Work is in progress to include domain-facilities in the package, i.e. to allow to depart from the default settings of REDUCE, for instance by using rational coefficient arithmetic.

The GENTRAN-Optimizer Interface, allows some additional preprocessing activities. We introduced the optional use of GENTRAN's declare-statement, thus allowing to specify the type of some or all of the lhs's and of the identifiers used to construct the rhs's. In addition to the prefixlist, a list of declarations in the Target Language can be produced, based on default assumptions conceming un-typed lhs's and identifiers in the input. This facility is based on the use of GENTRAN's symboltable.

Before optimizing rhs's it might be attractive to rewrite them using a generalized form of Horner's rule. We designed such a command, which does not necessarily have to be used in the context of the Optimizer. It can operate on a set of assignment statements and it can deliver the result in the form of a sequence of prefix-forms, defining the rewritten statements. Subjecting such a sequence of prefix-forms to an Optimizer-application implies that the GENTRAN-approach is not directly applicable. The GENTRAN := and :=: assignment operators define literal translation or ths-simplification, respectively. Therefore we extended our Interface with special facilities, allowing the Optimizer to accept the result of the application of such a command literally. Besides the g(eneralized)horner(rule) we have a command, generalizing the impact of the structr-command to a set of assignment statements. Similar possibilities exist for functions, when applied on their argument(s), give specific prefixform representations. Examples of such functions are the compression facilities, once made by Hulshof, during a stay at the Rand Corporation [13].

We discuss and illustrate a straightforward use of the Optimizer in section 2. In section 3 we introduce the special commands ghorner and gstructr and show how to use them in combination with the Optimizer. We use section 4 to discuss the declarationfacilities and section 5 to show the different file-handling possibilities. It is not our intention to give details of underlying algorithms. We merely want to survey the capabilities of the package in general terms. Section 6 is finally used to give some conclusions in summary and to indicate future work.

\section{Code Optimization : Strategy and Facilities}

\subsection{The Strategy}

Before illustrating the effect of applying the Optimizer, we shortly describe the operations, covered by the functions $T_{\beta}$ and $F$, mentioned in the previous section.

The function $\mathbf{R}$ accepts assigment statements given in prefixform. We can divide these forms in three categories using their leading operator. We distinguish between PLUS, TIMES and OTHER-operators. Leaving aside the OTHER-operators for awhile, we reduce the structure of possible rhs's to those of not necessarily expanded multivariate polynomials with integer coefficients. Assuming the leading operator is PLUS, the operands, being terms of a polynomial (for instance 
$\left.3 a+2 b+3 b^{2} c(3 a+2 b)(c+d)^{2}\right)$, can either be primitive or composite. A primitive term is an integer, an identifier or the product of an integer and an identifier. Hence the primitive terms of a sum form an (eventually empty) linear expression $(3 a+2 b)$. Composite terms are products, which cannot be qualified as a primitive term $\left(3 b^{2} c(3 a+2 b)(c+d)^{2}\right)$. Like sums, prefix-forms with a TIMES-operator, can have a primitive and/or composite part. The primitive part of a product is an (eventually empty) power product $\left(b^{2} c\right)$. The composite part is a product of sums and/or powers of sums $\left((3 a+2 b)(c+d)^{2}\right)$. Observe that our expression-structure discussed so far is still too simple. Powers of sums have EXPT as their leading operator $\left((c+d)^{2}\right)$. Similarly, a product can have a integer coefficient $\left(3 b^{2} c\right)$.

This description suggests, as already indicated in section 1, that we can consider any set of rhs's as being built with linear expressions and power products only. This allows to map such a set onto two incidence matrices: One defining the linear expressions, using the coefficients, and another defining the power products, using the exponents. The rows of these matrices can be associated with the (sub)expressions under consideration and the columns with the identifiers, used to construct these expressions. This is why we need to assume the validity of the commutative and associative law. To be able to retrieve the structure of the assignment statements forming the input set, we need to combine additional information with the rows and columns of these matrices. Essential is, for instance, storage of the exponents of sums and of the coefficients of products. Equally important is storage of the lhs's, which are the rhs-recognizers. Details are given in [15]. The examples 2.2 .1 and 2.2.2 provide illustrations of these data structures.

When introducing kemels, i.e. when assuming the set of OTHER-operators to be not empty, we have to store lists of non-commutable arguments. Therefore a table of pairs is made, formed by the kernels and their internally created names. These names are entered in the matrices as new identifiers. The arguments of such a kernel can be arbitrary REDUCE-expressions, which also have to be incorporated in the matrices. Hence the table is created recursively.

What is a cse and how do we locate its occurrences? A (sub)expression is common when it occurs repeatedly in the input. The occurrences are, as part of the input, distributed over the matrices, and shown as equivalent integer (sub)patterns. In fact, we repeatedly search for completely dense (sub)matrices of rank 1. The expression $2 a+3 c$ is a cse of $e_{1}=2 a+4 b+3 c$ and $e_{2}=4 a+6 c+5 d$, representable by $(2,4,3,0)$ and $(4,0,6,5)$, respectively. We indeed have to assume commutativity, so as to be able to produce new patterns $(2,0,3,0,0),(0,4,0,0,1)$ and $(0,0,0,5,2), \quad$ representing $s=2 a+3 c, \quad e_{1}=4 b+s \quad$ and $e_{2}=5 d+2 s$, respectively, and thus saving one addition and one multiplication. Such an additive cse can be a factor in a composite (sub)product, which in num can be reduced to a primitive product, when the cse is replaced by a new symbol. Therefore an essential part of an optimization step is regrouping of information. This migration of information between the matrices is performed if the Breuer-searches are temporarily completed. After this regrouping the distributive law is applied, eventually also leading to a further regrouping. If at least one of these actions leads to a rearragnment of information the function $T_{\beta}$ is again applied. Observe that this $\mathrm{T}_{\beta}$ is also a composite function. In view of the iterative character of the optimization process we always accept minimal profits.

A similar search is performed to detect multiplicative cse's, for instance occuring in $e_{1}=a^{2} b^{4} c^{3}$ and $e_{2}=a^{4} c^{6} d^{5}$. However, given a power product $\prod_{i=1}^{m} x_{i}^{a_{1}}$, any product $\prod_{i=1}^{m} x_{i}^{b_{1}}$, such that some $b_{i}<a_{i}$, for $\mathrm{i}=1(1) \mathrm{m}$, can function as a cse. We therefore extend the search for multiplicative cse's by employing this property, and as indicated in [15].

The function $F$-defining the finishing touch-performs one-row and/or one-column searches. Once the extended Breuer-searches do not lead to further reduction in the arithmetic complexity we try -applying it- to improve what is left. The integer coefficients in (sub)sums can have, possibly locally, a gcd, which can be factored out. One-column operations serve to discover and properly replace integer multiples of identifiers. As part of the outputprocess we subject all exponentiations left - at most one for each identifier - to an addition chain algorithm. Another action, covered by $F$ is therefore replacement by a new symbol of those (sub)sums, which are raised to some integer power.

\subsection{The Facilities}

REDUCE allows, roughly speaking, two modes of operation: ON EXP or OFF EXP. The first alternative is the default setting leading to expanded forms. The latter gives unexpanded forms, as discussed by Hearn in some detail $[3,4]$. It is obvious that the OFF EXP setting is in general preferable over the ON EXP setting when attempting to optimize the description of a set of assignment statements.

Starting a REDUCE session gives the initial state. All switches have their initial value: ON EXP, PERIOD and OFF FORT, for instance. When loading the Optimizer we create a new operating environment, without disturbing the current state of the system.

The result of an application of the Optimizer can be influenced by the use of certain REDUCE- or Optimizer-switches. The influence of EXP is obvious. By default the switch ACINFO is turned on. This guarantees an echo of the form in which the assignment statements are consumed by the Optimizer. It also guarantees the printing of tables with the numbers of arithmetic operations, occuring in $\mathrm{E}_{0}$ and $\mathrm{E}_{\lambda}$, respectively. Some switches are available to obtain information about the process itself. They were mainly introduced to assist in debugging and testing. PRIMAT for instance, can be used to vizualize both $D_{0}$ and $D_{\lambda}$. Output is by default given in REDUCE syntax, but FORTRAN syntax is possible in the usual way. The switch PREFIX can be used to obtain the prefixlist itself as output. This list can be used in the GENTRAN context to translate the output in a desired target language.

An optimization action is easily performed. Either the command "optimize <object>;" or the command "optimize <object> iname <cse-prefix>;" suffices. The <object> to be eleborated is either one assignment statement or a list of such statements, all obeying the GENTRAN rules. The <cse-prefix > is an identifier, used to generate the cse-names, by extending it with an integer part. The gensym-function is applied when the iname-extension is omitted.

We now illustate the use of the Optimizer through some small examples, by showing parts of REDUCE sessions.

\section{Example 2.2.1}

The multivariate polynomial $Z$ is a sum of 6 composite terms. These terms, monomials, are constant multiples of primitive products. A picture of $D_{0}$ is shown after the input echo. The sumsmatrix consists of only one row, identifiable by its $\mathrm{Fa}($ the)r $\mathrm{Z}$, the lhs. Its exponent is given in the $\mathrm{E}$ (xponent or ) $\mathrm{C}$ (oefficient) field. The 6 monomials are stored in the products-matrix. The coefficients are stored in the EC-fields and the predecessor row index, 0 , is given in the Far-field. Before the $D_{\lambda}$ picture is given the effect of the optimization process, the output and the opera- 
tor counts are shown. The optimized form of $Z$ is obtained by applying the distributive law. The output also shows applications of an addition chain algorithm ([18], 441466) as part of $R^{-1}$. although its use in example 2.2.3 is more apparent.

Observe that the output illustrates the heuristic character of the optimization process: In this particular case the ths can be written as a polynomial in $\mathbf{S}$, thus saving one extra multiplication.

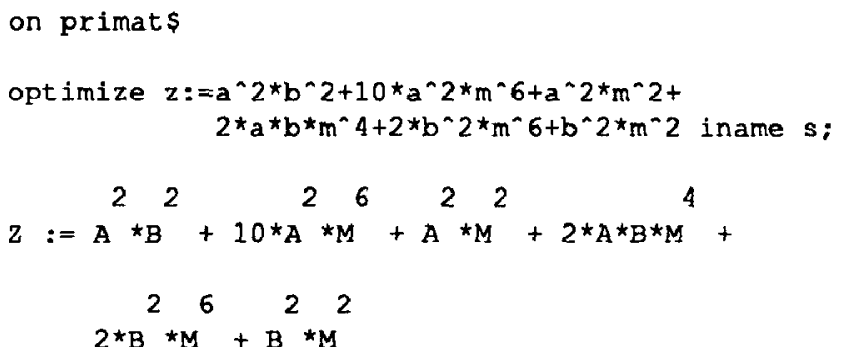

\begin{tabular}{|c|c|c|c|c|c|}
\hline 11 & & 2 & 21 & & 0 \\
\hline 21 & 6 & & 21 & $10 !$ & 0 \\
\hline 31 & 2 & & 21 & 11 & 0 \\
\hline 41 & 4 & 1 & 11 & 21 & 0 \\
\hline 51 & 6 & 2 & 1 & 21 & 0 \\
\hline 61 & 2 & 2 & 1 & 11 & 0 \\
\hline
\end{tabular}

$0: M$

$1: B$

$2: A$

Number of operations in the input is:

Number of $(+,-)$-operations : 5

Number of (*)-operations : 10

Number of integer exponentiations : 11

Number of other operations : 0

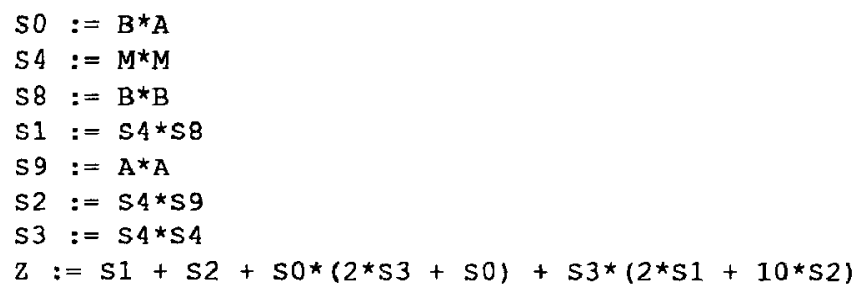

Sumscheme :

\begin{tabular}{rrrrrrr}
1 & 0 & 3 & 4 & 51 & ECIFar \\
\hdashline 01 & & & 1 & 11 & 11 & 2 \\
151 & & & 2 & 101 & 11 & 14 \\
171 & 2 & 1 & & 1 & 11 & 16 \\
\hdashline 0 & $:$ & 53 & & & & \\
3 & $:$ & 50 & & & & \\
4 & $:$ & 51 & & & & \\
5 & $:$ & 52 & & & &
\end{tabular}

Productscheme :

$$
\text { I } 891011171819201 \text { EC|Far }
$$

\begin{tabular}{|c|c|c|c|c|c|}
\hline 71 & & & 1 & 11 & 11 so \\
\hline 81 & 1 & & 2 & 1 & $1 \mid 51$ \\
\hline 91 & 1 & & & 21 & $1 \mid \leqslant 2$ \\
\hline 101 & 2 & & & 1 & 1153 \\
\hline 111 & & 2 & & 1 & $1 \mid 54$ \\
\hline 141 & 1 & & & 1 & 110 \\
\hline 161 & & 1 & & 1 & 110 \\
\hline
\end{tabular}

$8: 54$

$9: 53$

$10: 52$

$11: 51$

17 : so

$18: M$

$19: B$

$20: A$

\section{Example 2.2.2}

The input echo below shows the literal copy of the first assignment, in accordance with the GENTRAN := operator. The second assignment, again in accordance with the GENTRAN operator ::=:, has a rhs in expanded form.

The $\mathrm{D}_{0}$ picture shows that during parsing string matching of kernels in prefix-form already contributes to optimization : $S 2=$ $C^{*} X+D$ and S3 $=S I N(S 2)$ are stored once.

Application of the distributive law gives the original structure of $A(1,1)$ back.

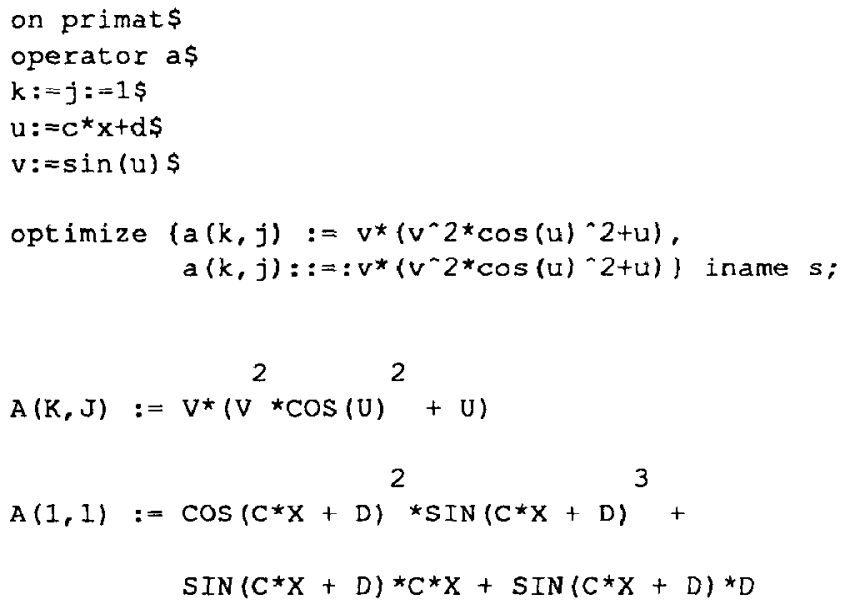


Sumscheme :

\begin{tabular}{rrrll}
1 & 7 & 81 & EC|Far \\
\hdashline 11 & 1 & 1 & 11 & 0 \\
$3 \mid$ & 1 & $1 \mid$ & A $(1,1)$ \\
51 & $1 \mid$ & $1 \mid$ & 52 \\
\hdashline 7 & $:$ & $U$ & & \\
8 & $:$ & 0
\end{tabular}

Product scheme :

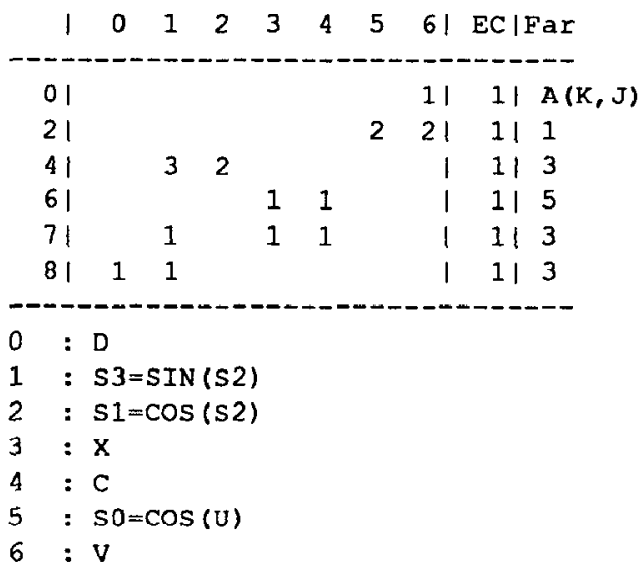

Number of operations in the input is:

Number of $(t,-)$-operations : 7

Number of $(*)$-operations : 10

Number of integer exponentiations : 4

Number of other operations : 5

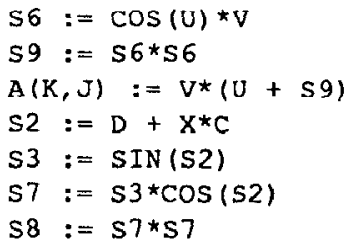

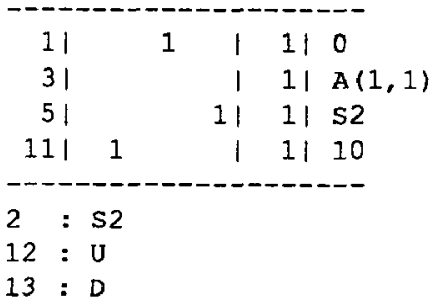

Product scheme :

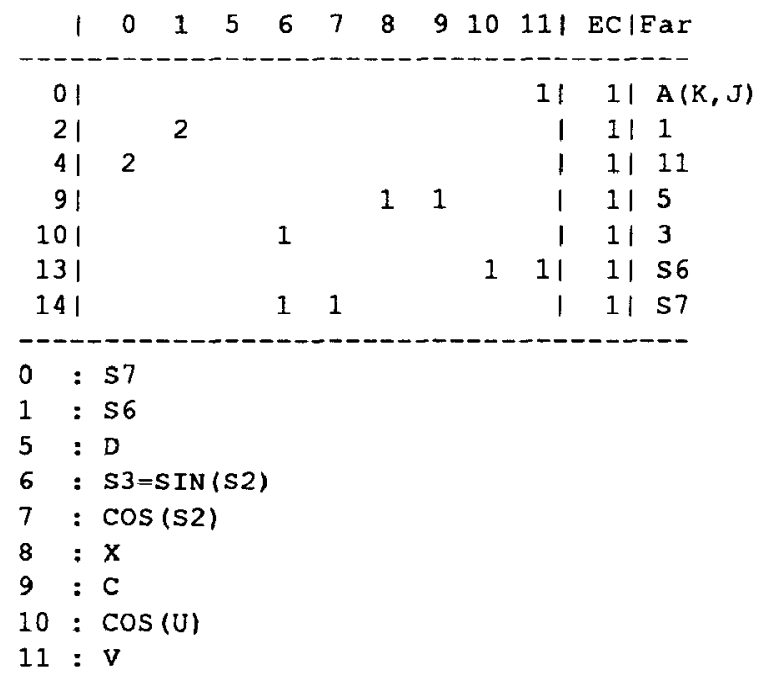

\section{Example 2.2.3}

The effect is shown of a finishing touch application, in combination with FORTRAN output. During output preparation $\mathrm{SO}^{13}$ is rewritten, using the earlier mentioned addition chain algorithm.

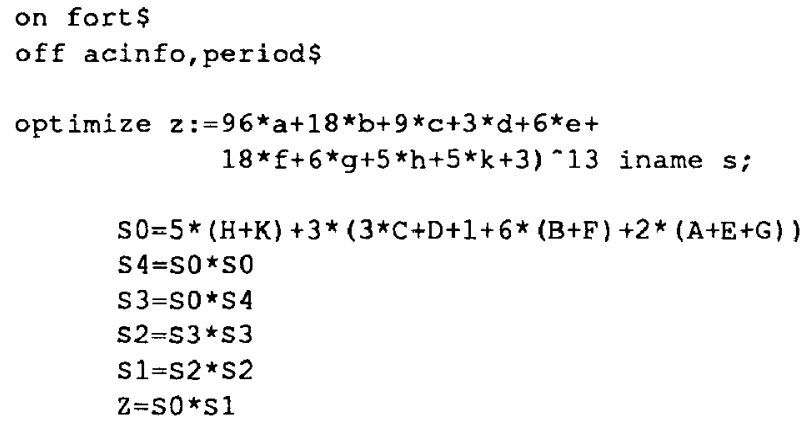

Example 2.2.4

Recovery of repeatedly occuring integer multiples of identifiers, as part of the finishing touch, is illustrated. The switch ACINFO is turned off.

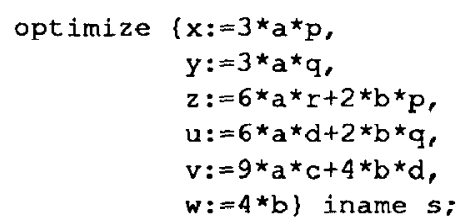




\section{Preprocessing Facilities}

It might happen that structure is obviously visible in the rhs's of a set of assignment statements, which we want to optimize. One can think of a set of partial derivatives of products. Or one might consider the application of Homer-rules. The first alternative demands for a generalized STRUCTR command. We implemented such a facility. Its syntax is straightforward: "gstructr <object> name <id>;" The <object> to be elaborated is one assignment statement or a set of such statements, separated by semicolons and grouped together between the special symbols $\ll$ and $\rightarrow$. In stead of a statement a matrix-name is also allowed. Then all non-zero matrix elements are incorporated in the search for obvious cse's. The <id> of the optional name-part, being an identifier, is used to identify the subexpressions, produced via the application of a gstructr command. When the switch ALGPRI is on -the default setting- the output is given in REDUCE syntax, while turning it off leads to output in prefixform. The latter is employed by the function $R$, the Optimizerparser. It is also possible to get FORTRAN-output by turning off the switch PERIOD and turning on the switch FORTRAN. The input remains unchanged when the switch EXP is on.

\section{Example 3.1}

We show part of a REDUCE session.

off exps

$\operatorname{matrix} a(2,2) \$$

$a(1,1):=x+y+z \$$

$a(1,2):=x^{\star} y \$$

$a(2,1):=(x+y) * x * y \$$

$a(2,2):=\left(x+2^{\star} y+3\right)^{-3} 3-x \$$

on fort\$

off periods

GSTRUCTR «A;

$A A:=(X+Y) \wedge 2$

$B:-(X+Y) *(Y+Z)$;

$C:=(X+2 * Y) \star(Y+Z) \star(Z+X) \wedge 2$

$\gg$ NAME $v_{i}$

$\mathrm{V} 1=\mathrm{X}+\mathrm{Y}+\mathrm{Z}$

$A(1,1)=V 1$

$A(1,2)=X^{\star} Y$

$\mathrm{V} 2=\mathrm{X}+\mathrm{Y}$

$A(2,1)=V 2 \star X * Y$

$\mathrm{V} 3=\mathrm{X}+2 \star \mathrm{Y}+3$

$\mathrm{V} 4=\mathrm{V} 3 * \star 3-\mathrm{X}$

$A(2,2)=V 4$

$\mathrm{AA}=\mathrm{V} 2 \star \star 2$

$\mathrm{V} 5=\mathrm{Y}+\mathrm{Z}$

$\mathrm{B}=\mathrm{V} 2 * \mathrm{~V} 5$

$\mathrm{V} 6=\mathrm{X}+2 * \mathrm{Y}$

$\mathrm{V} 7=\mathrm{X}+\mathrm{Z}$

$\mathrm{C}=\mathrm{V} 6 * \mathrm{~V} 7 * 2 * \mathrm{~V} 5$

The syntax for the ghorner-command is similar. The application of a Homer-rule assumes an ordering of the identifiers. We allow instead of the name-part of the gstructr command an optional vorder <list of id.s> extension. The <list of id.s> consists of at least one identifier. This list overrules, in the order given, the current identifier ordering of the system. The rhs's are considered as polynomials in the leftmost element of the vorder-list. The thus created coefficients are in turn considered as polynomials in the second element of this list. And so on. When the vorder-extension is omitted the current system identifier ordering is applied. The switch ALGPRI is again applicable and has the same meaning as for gstructr.

Some optimizing compilers apply Homer-rules when possible. Our optimization strategy is based on an all levels, all expressions search. This contradicts the Horner-mechanism. To avoid destabilizing side-effects of Horner-rule applications we decided to bring such a facility under user-control.

\section{Example 3.2}

Some Taylor-expansions are shown. The definition of the function Taylor is obvious.

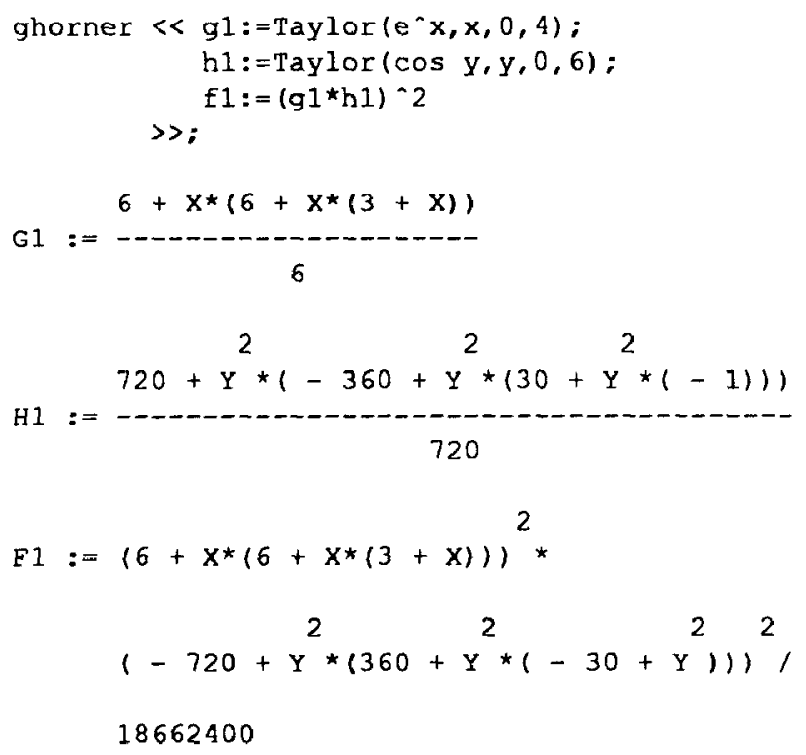

Both commands can be used inside an optimize-command.

\section{Example 3.3}

We show the effect of an applying the optimize command on the gstructr command of example 3.1. Observe that the cse-names produced by the optimizer begin with an $S$, while the gstructr created names start with a V.

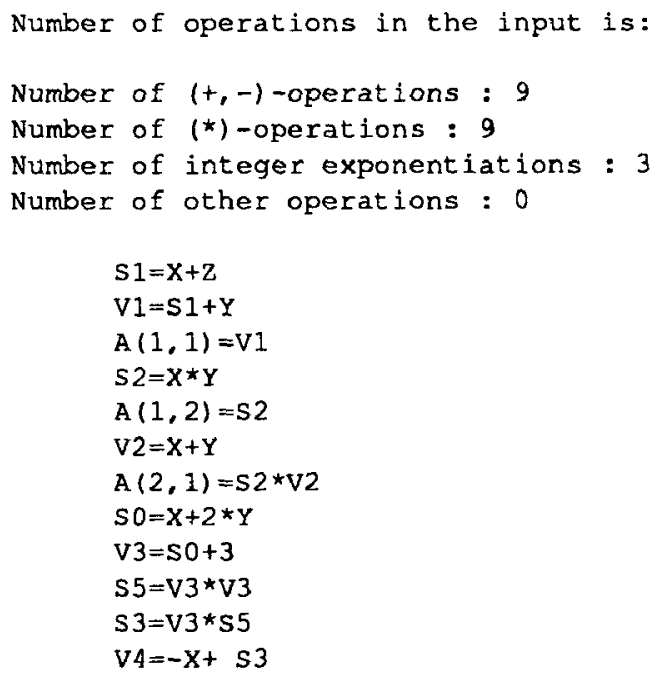




$$
\begin{aligned}
& \mathrm{A}(2,2)=\mathrm{V} 4 \\
& \mathrm{AA}=\mathrm{V} 2 * \mathrm{~V} 2 \\
& \mathrm{~V} 5=\mathrm{Y}+\mathrm{Z} \\
& \mathrm{B}=\mathrm{V} 2 * \mathrm{~V} 5 \\
& \mathrm{~V} 6=\mathrm{S} 0 \\
& \mathrm{~V} 7=\mathrm{S} 1 \\
& \mathrm{~S} 4=\mathrm{V} 7 * \mathrm{~V} 7 \\
& \mathrm{C}=\mathrm{V} 5 * \mathrm{~V} 6 * \mathrm{~S} 4
\end{aligned}
$$

Number of operations after optimization is:

Number of $(+,-)$-operations : 7

Number of (*)-operations : 11

Number of integer exponentiations : 0

Number of other operations : 0

\section{Generation of Declarations}

The GENTRAN declare-statement can also be used as one of the arguments of the optimize-command. An illustration of this facility is given in example 4.1. The syntax of such a statement is in accordance with the GENTRAN-rules [3]. We also use the symbol-table of GENTRAN. During parsing, the declared identifiers and/or array- and matrix-names are entered in the symbol-table. Once optimization is finished all relevant information for completing the declarations is known, and collected in prefixlist. The untyped identifiers and the system selected csenames are entered in the symbol-table, making use of already known information, expression-structure and the normal hierarchy in data types. Once this table is completed a list of declarations is produced if the switch OPTDECS is turned on. The output is by default given in REDUCE syntax. Alternative output is obtained by assigning a relevant value to the global identifier Optlang!*. This causes GENTRAN to take over the output preparation, as shown in:

\section{Example 4.1}

The initial declarations in the declare-part of the optimizecommand are missing a description of $Y$. An educated guess is made for the type of $Y$, while the array-length remains unspecified and is simply given by $\mathbf{N}$.

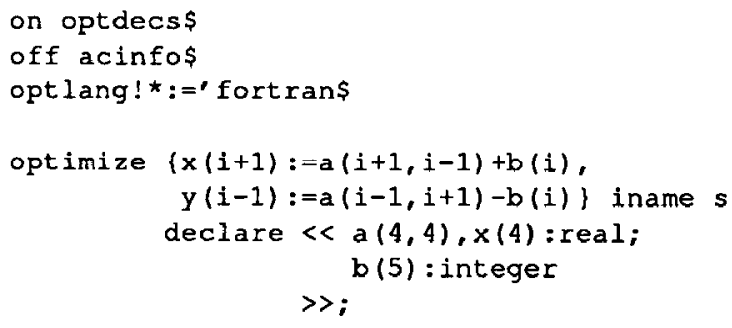

The C-parser of GENTRAN does not allow unspecified array bounds. This implies that the $C$-version of the above given piece of FORTRAN program, as shown below, can only be produced when the declare-part is completed with the declaration of $Y$.

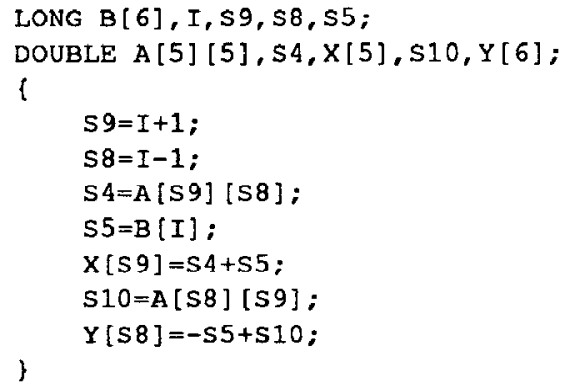

\section{File Management and Optimization Strategies}

Another possible optimize-argument is formed by the sequence in file $e_{1}$, file $e_{2}, \ldots$, file $_{n}$. Each of these files is assumed to contain one or more assignment statements, obeying the GENTRANassignment rules. An Optimizer application results in a unified sequence of assignment statements in the target language.

However, some switches are available for stepwise performing such an optimization task. Turning on the switch CRUNCH results in a suppression of the finishing touch and the output, although the prefixlist is made. This list is used, in combination with the input of the next optimize-call, to create a fresh version of $D_{0}$. Finally turning off this switch before the last optimizecommand to be performed, results in a unified output-sequence. It is also possible to continue with optimization of the potential set of input assignments during another session. When turning on the switch AGAIN, the system is instructed to save relevant internal information in combination with the result of the present optimization run. The thus extended output is assumed to be stored in a file. When the optimization task is continued during another session this file is assumed to be read before all other remaining files. The system is instructed to expect this file by turning on the switch REMEMBER.

\section{Some Conclusions and Future Work}

The present version of the Optimizer produces output in FORTRAN syntax, for instance. However, the target machine, and thus a specific (optimizing) compiler, are seemingly unknown. This is a disadvantage of source to source operations. The efficiency of the generated code is related to peculiarities of the computer in use. Application of the addition chain algorithm might for instance result in lots of small assignment statements, forcing to use many store instructions. Introducing weight functions in the form of parameters might help, assuming the optimization strategy itself is not affected. This strategy is based on stepwise restructuring of information. This means that rejecting intermediate low profit can spoil the optimization attempts. Hence we consider to eventually remodel the prefixlist, based on machine dependent weights.

Part of our research is dedicated to domain independent code optimization. This is especially interesting for simplification, as suggested by Heam [7,8]. However it requires, most probably, to re-introduce the standard-quotient-form parser, since such operations ought to take place below the user level and as closely related to the internal algebra as possible. 
Optimization thus far was limited to blocks of straightline code. We are also working on variants of our heuristic approach for vector- and parallel architectures (see for instance [5]). Part of this research is dedicated to automated data flow and data dependences analysis [10]. We intend to combine such features with the Optimizer, thus creating an environment to perform global optimization as well. An obvious application is a refinement of our code for computing Jacobians and Hessians [11].

\section{Availability}

The Optimizer will be added to the REDUCE Library when integration with some other facilities for improvement of output presentation and the manual are completed.

\section{Acknowledgements}

The many discussions we had over the past years with Anthony C. Hearn and Paul S. Wang about the symbolic-numeric aspects of computer algebra have been very stimulating and valuable. The work of one of us (BLG) was supported by The National Science Foundation under Grant No. ASC-8516384.

\section{References}

[1] Aho, A.V., Sethi, R., Ullman, J.D.: Compilers Principles, Techniques and Taols. Reading, Mass.: Addison-Wesley (1986).

[2] Breuer, M.A.: "Generation of optimal code for expressions via factorization", Comm. $A C M$ 12,6, 330-340 (1969).

[3] Gates, B.L.: "GENTRAN: An automatic code generation facility for REDUCE", A.C.M. SIGSAM Bulletin 19,3, 24-42. New York: A.C.M. (1985).

[4] Gates, B.L., Wang,P.S.: "A LISP-based RATFOR code generator", Proceedings 1984 MACSYMA User's Conference (V.E. Golden, ed.), 319-329. Schenectady, N.Y.: Gen. El. (1984).

[5] Goldman, V.V., van Hulzen, J.A.: "Automatic code vectorization of arithmetic expressions by bottom-up structure recognition", Proceedings CAP '88 (E. Toumier, ed.), (to appear).

[6] Gonzales, T., Ja' Ja', J.: "Evaluation of arithmetic expressions with algebraic identities", SIAM J. Comp., 11, 4, 633-662 (1982).

[7] Hearn, A.C.: "Structure: The key to improved algebraic computation", Proceedings RSYMSAC2 (N. Inada en T. Soma, ed.'s), 215-230. Singapore: World Scientific Publ. (1985).

[8] Heam, A.C.: "Optimal evaluation of algebraic expressions", Proceedings AAECC-3 (J. Calmet, ed.), Springer LNCS series nr 229, 392-403. Heidelberg: Springer Verlag (1986).

[9] Hearn, A.C.: REDUCE user's manual, version 3.3. Santa Monica, CA: The Rand Corporation (1987).

[10] van Heerwaarden, M.C.: Data dependences analysis for program generation and optimization, Masters Thesis, University Twente, Dept. of Computer Science (june 1989).
[11] van den Heuvel, P., van Hulzen, J.A., Goldman, V.V. "Automatic generation of FORTRAN-coded Jacobians and Hessians", Proceedings EUROCAL '87 (J.H. Davenport, ed.), (to appear).

[12] Hulshof, B.J.A., van Hulzen, J.A.: "Automatic error cumulation control", Proceedings EUROSAM '84 (J.P. Fitch, ed.), Springer LNCS-series 174, 260-271. Heidelberg: Springer Verlag (1984).

[13] Hulshof, B.J.A., van Hulzen, J.A.: "An expression compression package for REDUCE based on factorization and controlled expansion", Proceedings EUROCAL "85, Vol. 2 (B.F. Caviness, ed.), Springer LNCS series nr 204, 315-316. Heidelberg: Springer Verlag (1985).

[14] van Hulzen, J.A.: "Breuer's grow factor algorithm in computer algebra", Proceedings SYMSAC ' 81 (P.S. Wang, ed.), 100-104. New York: A.C.M. (1981).

[15] van Hulzen, J.A.: "Code optimization of multivariate polynomial schemes: A pragmatic approach", Proceedings EUROCAL '83, (J.A. van Hulzen, ed.), Springer LNCS series 162, 286-300. Heidelberg: Springer Verlag (1983).

[16] Johnson, D.B., Miller, W., Minnihan, B., Wrathall, C.: "Reducibility among floating-point graphs", J. ACM 26, 4, 739-760 (1979).

[17] Knuth, D.E.: "An empirical study of Fortran programs", Software Practice and Experience 1, 105-133 (1971).

[18] Knuth, D.E.: The art of computer programming, Vol. 2 (Second Edition). Reading, Mass.: Addison-Wesley (1980).

[19] Molenkamp, J.H.J., Mellink, J.; Some investigations concerning symbolic-numeric strategies in linear programming, Masters Thesis (in Dutch), University Twente, Dept. of Computer Science (1988).

[20] Smit, J., van Hulzen, J.A., Hulshof, B.J.A.: "NETFORM and code optimizer manual", A.C.M. SIGSAM Bulletin 15,4, 23-32. New York: A.C.M. (1981).

[21] Smit, J., van Hulzen, J.A.: "Symbolic-numeric methods in microwave technology", Proceedings EUROCAM '82 (J. Calmet, ed.), Springer LNCS series 144, 281-288. Heidelberg: Springer Verlag (1982).

[22] Wang, P.S., Chang, T.Y.P., van Hulzen, J.A.: "Code generation and optimization for finite element analysis", Proceedings EUROSAM '84 , (J.P. Fitch ed.), Springer LNCS series 174, 237-247. Heidelberg: Springer Verlag (1984). 